\title{
Molecular characterization of regulatory polymorphisms in the promoter region of the STAT6 gene in a Gabonese population
}

\author{
Thirumalaisamy Palanichamy Velavan ${ }^{1 /+}$, Silke Bechlars ${ }^{1}$, Jürgen Tomiuk², \\ Peter G Kremsner1,3, Jürgen FJ Kun' \\ ${ }^{1}$ Institute for Tropical Medicine ${ }^{2}$ Institute for Human Genetics, University of Tübingen, Tübingen, Germany \\ ${ }^{3}$ Medical Research Unit, Albert Schweitzer Hospital, Lambaréné, Gabon
}

\begin{abstract}
Parasites remain competent invaders of host immunity. Their invasion strategies have proven to impact immunorelevant genes leading to diversity among gene families. We focussed on signal transducer and activator of transcription (STAT6) factor that plays a fundamental role in signal transduction and activation of transcription. Recent studies have highlighted the role of STAT6 variants in control of infection levels. We identified and investigated regulatory single nucleotide polymorphisms (SNPS) in the promoter regions of the STAT6 gene in a group of Gabonese individuals exposed to a variety of parasitic infections. Three promoter variants were identified in 40 individual subjects. We further validated these promoter variants for their allelic gene expression using transient transfection assays. One promoter variant, $r s 3024944(G / C)$, revealed an altered expression of the marker gene. The identification of functionaltering SNPs in the promoter may facilitate studying parasite susceptibility in association studies.
\end{abstract}

Key words: STAT6 - polymorphism - transfection - regulatory T cells

Human host counteracts a wide array of parasitic diseases; however, the crucial aspect is the failure of the host immune system to clear these parasites despite antigen recognition. The underlying presumption is that immunomodulation by parasites induces regulatory $\mathrm{T}$ cells, which, in turn, suppress anti-parasite effector cells, leading to a diffused immune response. Regulatory T cells (Tregs) are defined as a "self-check" mechanism to inhibit autoimmune reactions, which develop naturally in both humans and mice (Sakaguchi 2000, 2004, 2005). Several types of regulatory cells exist; some are induced in response to infectious challenge and the others are considered natural regulators (Belkaid \& Rouse 2005). Investigation of human polymorphisms in the promoter region of immune genes will possibly reflect the level of susceptibility to parasitic infections and of Treg expression (Belkaid \& Rouse 2005). Gene expression is instigated by an ordered transcriptional process in the promoter region of the gene and is regulated by various types of $c i s$-acting DNA sequence elements located at the promoter region. Consequently, polymorphisms in the promoter regions (regulatory polymorphisms) can direct substantial changes in gene expression. One such gene of interest that plays a key role in altering susceptibility to a parasitic infection is the signal transducer and activator of transcription 6 (STAT6) gene locus.

The STAT6 gene belongs to the STAT family of transcription factors and is believed to be responsible for mediating various cytokine-induced responses. In general, STATs play a role in signal transduction and in the activation of

Financial support: EU-TRANCHI (INCO-CT-2006-032436)

VTP and SB equally contributed to the work.

+ Corresponding author: velavan@medizin.uni-tuebingen.de

Received 25 June 2010

Accepted 12 November 2010 transcription. In the cytoplasm, these STAT proteins exist in an inactive form and are phosphorylated by the Janus kinase family of tyrosine kinases following cytokine-receptor interactions (Kaplan et al. 1996). On phosphorylation, these proteins form dimers then translocate to the nucleus and bind to defined DNA sequences to regulate gene transcription (Schindler \& Darnell 1995). STAT6 proteins are particularly involved in the interleukin-4 signalling pathway, where they are activated by stimulation of IL4 and IL13 (Kaplan et al. 1996). Recent studies have demonstrated that STAT6 gene promoter polymorphisms are associated with high infection levels in urinary schistosomiasis. In particular, one promoter polymorphism (rs324013) in STAT6 was found to be associated with the control of infection levels (He et al. 2008). Similarly, a STAT6 variant (rs324015) was associated with resistance to Ascaris in children from an endemic area in China (Peisong et al. 2004). A very recent study in the context of active inflammation in asthma patients (Pillemer et al. 2009) concluded that blocking the dominant stimulatory effects of IL4 and IL13 via STAT6 on disease-associated Th2 cells is expected to result in a beneficial effect in asthma patients.

In the current study, we aim to identify regulatory single nucleotide polymorphisms (SNPs) on the STAT6 gene locus from 40 unrelated Gabonese individuals who had earlier infection episodes with various parasites. These identified regulatory SNPs that are pre-described in the database were further validated for their allelic gene expression, which may possibly correlate with various physiological responses.

\section{SUBJECTS, MATERIALS AND METHODS}

Genomic DNA isolation - A total of 40 DNA samples obtained from unrelated Gabonese individuals were used to identify SNPs in the promoter region of STAT6 genes by direct sequencing. Blood samples were collected from adult patients with uncomplicated malaria at the 
Medical Research Unit of the Albert Schweitzer Hospital (Lambaréné, Gabon) between August-November 2004. The study was approved by the local ethical committee of the International Foundation of the Albert Schweitzer Hospital. DNA was isolated using the QIAamp DNABlood Mini Kit (Qiagen, Hilden, Germany).

Sequencing and SNP identification - For sequencing analysis, gene and genomic sequences (NM003153) were obtained from the SNPper database (http://snpper. chip.org/) and polymerase chain reaction (PCR) primers were designed using the PRIMER3 software (Rozen \& Skaletsky 2000) (www-genome.wi.mit.edu/cgi-bin/ primer/primer3 www.cgi). Primers were designed to amplify the promoter regions of the STAT6 gene. Fragments of the promoter of the STAT6 gene were amplified by PCR using the primer - STAT6-forward (5'-TTGTGGTCAGGGAAGTTGTG-3'), STAT6-intern01 (5'-GGACCTAGGAGTTGGCTGGCATCGAG-3'), intern02 (5'-CCCCAGTCCTGATCCCCCACGTG-3') and with STAT6-reverse (5'-CATGCACTCATAGAGGCCC-3') (MWG Operon, Ebersberg, Germany). The promoter region of the STAT6 gene was amplified using STAT6-forward and STAT6-reverse primer pairs in a singleplex reaction for each sample. The amplified promoter region is $764 \mathrm{bp}$ upstream from the transcriptional starting point. PCR amplifications were carried out in $20 \mu \mathrm{L}$ reaction volumes with $5 \mathrm{ng}$ of genomic DNA, $1 \mathrm{x}$ PCR buffer $(20 \mathrm{mM}$ Tris- $\mathrm{HCl}$ $\mathrm{pH}$ 8.4, $50 \mathrm{mM} \mathrm{KCl}, 1.5 \mathrm{mM}$ of $\mathrm{MgCl}_{2}$; Qiagen), 0.125 $\mathrm{mM}$ of dNTPs, $0.5 \mathrm{mM}$ of each primer and $1 \mathrm{U}$ Taq DNA polymerase (Qiagen, Hilden, Germany) on a PTC-200 Thermal Cycler (MJ Research, USA). Thermal cycling parameters were as follows: initial denaturation at $94^{\circ} \mathrm{C}$ for $2 \mathrm{~min}$, followed by 40 cycles of $30 \mathrm{~s}$ at $94^{\circ} \mathrm{C}$ denaturation, $30 \mathrm{~s}$ at $64^{\circ} \mathrm{C}$ annealing temperature, $1 \mathrm{~min} 30 \mathrm{~s}$ at $72^{\circ} \mathrm{C}$ extension, followed by a final extension of $2 \mathrm{~min}$ at $72^{\circ} \mathrm{C}$. PCR products were cleaned using Exo-SAP-IT (USB, Affymetrix, USA) and $1 \mu \mathrm{L}$ of the purified product were directly used as templates for sequencing, using the BigDye Terminator v.2.0 Cycle Sequencing Kit (Applied Biosystems, USA) on an ABI 3100 DNA Sequencer, according to the manufacturer's instructions. The primers employed for sequencing PCR were STAT6-forward, STAT6-intern02 and STAT6-reverse. DNA polymorphisms were identified when assembled with reference sequence of the STAT6 gene (NM003153) on chromosome 12 (position chr12:55,775,462-55,791,428) obtained from SNPper database (http://snpper.chip.org/) using the BioEdit (www.mbio.ncsu.edu/BioEdit/bioedit.html) and Seqscape v.2.5 (Applied Biosystems, USA) program.

Cloning and construct preparation - To confirm that the SNPs were without PCR errors introduced by the Taq polymerase, the respective genomic DNA identified to have SNPs was amplified with primers containing a $15 \mathrm{bp}$ homology to the linearized vector and were cloned into a pGL3 basic vector. In brief, PCR amplifications were carried out in $50 \mu \mathrm{L}$ reaction volumes with the same program conditions as previously mentioned. The amplified PCR products were analyzed by electrophoresis in $1.5 \%$ agarose gels, with a 100 bp DNA ladder molecular size marker (Invitrogen, Karlsruhe, Germany). PCR products were eluted from the gel and purified using a Nucleospin Kit (Macherey-Nagel, Düren, Germany). Cloning was done into the pGL3 basic vector using the In-Fusion Advantage PCR Cloning Kit (Clontech, Mountain View, CA). Plasmids were isolated using QIAprep ${ }^{\circledR}$ Spin Miniprep Kit (Qiagen, Hilden, Germany). To ensure accuracy of the sequenced promoter regions, several independent plasmids containing inserts were sequenced in both directions using appropriate primer pairs. The plasmid that exhibited true polymorphism was transformed into one shot Escherichia coli (Invitrogen, Karlsruhe, Germany). Two independent colonies were picked from these transformations and DNA was prepared using an EndoFree Plasmid Maxi Kit (Qiagen, Hilden, Germany).

Transient transfection assays - We tested the activities of the observed polymorphic promoters using Jurkat $\mathrm{T}$ cell lines. In principle, four independent transfection experiments for each construct in duplicates were performed with Jurkat T cells. Jurkat T cells $\left(0.8 \times 10^{6}\right.$ cells $\left./ \mu \mathrm{L}\right)$ were grown in a Roswell Park Memorial Institute (RPMI) 1640-supplemented medium (Sigma-Aldrich, Hamburg, Germany) containing $10 \%$ fetal bovine serum, $2 \mathrm{mM} \mathrm{L}$-glutamine and $1 \%$ streptomycin-penicillin substrate (Invitrogen, Karlsruhe, Germany). Jurkat cells $\left(0.8 \times 10^{6}\right.$ cells $\left./ \mu \mathrm{L}\right)$ were transfected with TransIT reagent (Mirus Bio, Madison, USA) as recommended by the manufacturer. In brief, $120 \mu \mathrm{L}$ of TransIT reagent was added to $3 \mathrm{~mL}$ of RPMI 1640 serum free medium (Sigma-Aldrich, Hamburg Germany) and was incubated for $20 \mathrm{~min}$ at room temperature (RT). Each of the 24-well plates was then seeded with $0.5 \mathrm{~mL}\left(0.8 \times 10^{6}\right.$ cells/ $\mu \mathrm{L}$ ) of Jurkat T cells along with $500 \mathrm{ng}$ of plasmid DNA constructs in addition to $20 \mathrm{ng}$ of Renilla and was allowed to incubate for $20 \mathrm{~min}$. After being incubated, $52 \mu \mathrm{L}$ of TransIT + RPMI serum-free medium mix were suspended across each well. The whole procedure was performed in 2 x 24-well plate formats. After $24 \mathrm{~h}$, one plate was induced with $20 \mathrm{ng} / \mathrm{mL}$ Phorbol 12-myristate 13-acetate (PMA, Sigma-Aldrich, Hamburg, Germany) and with $25 \mu \mathrm{g} / \mathrm{mL}$ Concanavalin A (Sigma-Aldrich, Hamburg, Germany). After $24 \mathrm{~h}$, cells were harvested by centrifugation, washed twice with phosphate-buffered saline and lysed in $100 \mu \mathrm{L}$ of $1 \mathrm{x}$ passive lysis buffer (Promega, Mannheim, Germany). After incubation for $20 \mathrm{~min}$ at RT on a rocking platform, $10 \mu \mathrm{L}$ of the lysate was used for the measurement of luciferase activity in the SIRIUS Luminometer (Berthold detection system, Pforzheim, Germany). The experiments are performed using dual luciferase reporter assay system (Promega, Mannheim, Germany). For each experiment, a constitutively expressed Renilla luciferase in low amounts was used as positive control (Jüliger et al. 2003); a promoterless plasmid (pGL3 basic) was integrated as a negative control. Each construct was measured eight times, stimulated and non-stimulated with two different DNA preparations. Relative luciferase activity was calculated as luciferase firefly/ luciferase Renilla multiplied by 1,000.

Transcription factor binding search - An extensive search for transcription factor-binding sites for the observed SNPs in the promoter region was performed using TF-Search online tool (www.cbrc.jp/research/ db/TFSEARCH.html). 
TABLE I

Genetic variants identified in the promoter regions of the signal transducer and activator of transcription 6 gene locus

Single nucleotide

polymorphisms

(rs\#)

Polymorphism Flanking sequences

Analyzed

\begin{tabular}{llllllll}
\hline rs3024941 & C $>$ G & GCAGG[C/G]AGCAG & CC & 33 & C & 0.825 & 0.858 \\
rs3024943 & & & GC & 7 & G & 0.175 & 0.142 \\
& G $>$ A & CCTGG[G/A]GAGCC & GG & 34 & G & 0.85 & 0.820 \\
rs3024944 & & AG & 6 & A & 0.15 & 0.180 \\
& \multirow{2}{*}{ G $>$ C } & \multirow{2}{*}{ GCAGA[G/C]TTTGA } & GG & 37 & G & 0.925 & 0.905 \\
& & & CG & 3 & C & 0.075 & 0.095 \\
\hline
\end{tabular}

$a$ : frequency corresponds for both allele and genotypes; $b$ : Yoruba population representing sub-Saharan African individual group.

Statistical analysis - Data have been analyzed by StatView (www.statview.com). Comparison of the luciferase activity of the three different STAT6 promoter variants was analyzed by a t-test (before and after stimulation). In addition, each construct's activity was compared to the activity of the wild type (common alleles) at both the induced and non-induced states. The statistically significant level was set as $>0.05$. Haplotype analysis was performed using the Haploview v.4.2 software (Barrett et al. 2005).

\section{RESULTS}

All 40 subjects were sequenced for the promoter region and were analyzed for SNPs. In total, three SNPs were identified in 40 subjects, all of which had already SNP IDs: rs3024941, rs3024943 and rs3024944. Their respective genotype and allelic frequencies are summarized in Table I. All three of the observed SNPs were heterozygous. Construct 2 possessed two SNPs, $\mathrm{C} / \mathrm{G}$ and $\mathrm{G} / \mathrm{A}$, which are linked. Four out of eight possible haplotypes were found. The number of haplotypes observed and their corresponding frequencies is represented (Table II). Based on haplotype frequencies, the degree of expected heterozygosity across three loci was 0.227 , which corresponds closely to the observed heterozygosity, which is 0.250 . The comparison of linkage disequilibrium between three loci in the promoter regions of the STAT6 gene locus as inferred from Haploview is represented in Fig. 1. Comparison of the activity of the three different STAT6 promoter variants analyzed by luciferase activity is depicted in the Fig. 2. All four constructs could be induced by phorbol myristate acetate/concanavalin A significantly ( $\mathrm{p}$ $<0.0001$ ) when compared before and after induction. The construct 3 variant had a higher luciferase activity in comparison to other two variants and remained significant in comparison to the wild type both at induced and non-induced state $(\mathrm{p}=0.003 ; \mathrm{p}=0.016)$. The comparison of expression levels of the reporter gene between the wild-type alleles and construct 1 and 2 did not show statistically significant differences $(\mathrm{p} \geq 0.50)$. The TF-Search online tool indicated that the SNP in
TABLE II

Observed haplotypes in the promoter regions of the signal transducer and activator of transcription 6 gene locus

\begin{tabular}{lcc}
\hline Haplotypes & $\begin{array}{c}\text { Expected } \\
\text { numbers }\end{array}$ & Frequency \\
\hline CGG & 70 & 0.875 \\
GGG & 1 & 0.012 \\
GAG & 6 & 0.075 \\
CGC & 3 & 0.038 \\
\hline
\end{tabular}

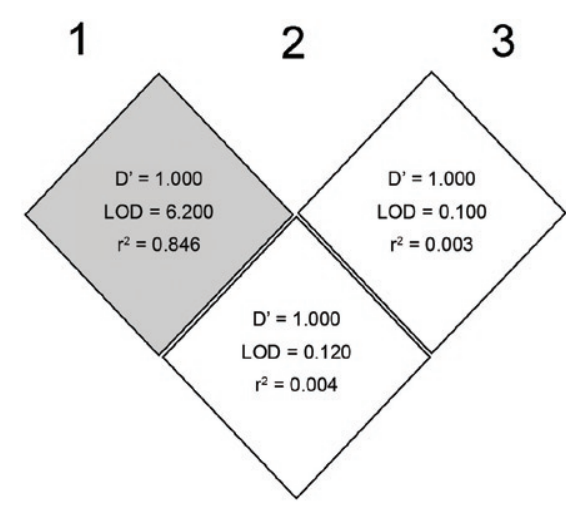

Fig. 1: comparison of linkage disequilibrium between three loci in the promoter regions of the signal transducer and activator of transcription 6 gene locus. D': value of D prime between two loci; LOD: likelihood odds ratio; $\mathrm{r}^{2}$ : correlation coefficient between two loci.

construct 2 (G/A \#rs3024943) is located in a putative transcription factor site myeloid zinc finger-1 (MZF-1). The SNP in construct 1 (C/G \#rs3024941) does not alter putative transcription factor binding sites when examined, whereas the SNP variant in construct $3(\mathrm{G} / \mathrm{C}$ \#rs3024944) is located in a transcription factor binding site for the T cell factor-1 (TCF-1). 


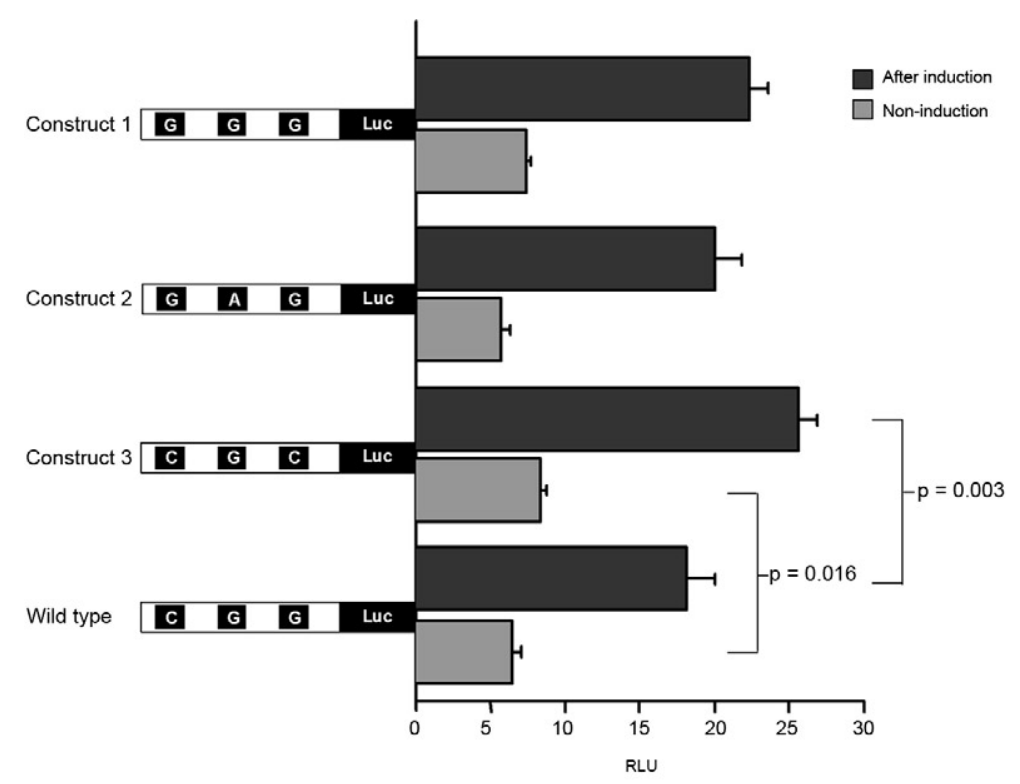

Fig. 2: comparison of the activity of the three different signal transducer and activator of transcription 6 promoter variants analyzed by luciferase activities. Given is the ratio of the relative light units (RLU) (firefly/renilla). p values are calculated by t-test. Each construct induced was with phorbol myristate acetate and concanavalin A. The letters indicate the presence of mutations G/C, G/A and C/G.

\section{DISCUSSION}

The critical barrier in control of infections remains to be the failure of the immune system to clear parasites, despite antigen recognition. Parasites represent a major selective force by influencing the genetic predisposition determined by immune system gene variants (Maizels 2009). Variations in the promoter regions of these immune system genes potentially modify the gene expression levels by changing specificity of transcription binding sites and/or by altering the kinetics of transcription initiation. In our current study, we identified three pre-described SNP variants in the studied Gabonese population. When compared to the National Center for Biotechnology Information HapMap database, all three observed SNP variants were found in the Yoruba population of Nigeria representing a sub-Saharan African group. The allele frequencies described in the HapMap database corroborate with allele frequencies observed in this study (Table I). A very large cohort will remain obligatory to associate any disease severity with these identified mutations. Interestingly, we found the promoter variant rs3024944 (G/C) exhibited an altered expression of the marker gene when compared to that of the wild type at a basal level and after induction. However, we did not find a difference in expression between the other two constructs compared to the wild type. The SNP in construct 2 (G/A \#rs3024943) is located in a transcription factor binding site MZF-1. MZF-1 is a Krupple class zinc finger gene encoding a putative transcriptional regulator of myeloid differentiation. MZF-1 is expressed in nonhaematopoietic cells as a transcriptional repressor, whereas in haematopoietic cells it acts as a transcriptional activator (Morris et al. 1995). However, a study concluded that there was no expression of MZF-1 expression levels in Jurkat T cells (Hromas et al. 1991). Therefore the SNPs in this binding site do not lead to altered expression levels. Construct 2 (G/A \#rs3024943) was linked to SNP variant (C/G \#rs3024941). Although linked, the effect of MZF-1 expression levels in Jurkat T cells might be a lone contribution by construct 2 because construct 1 does not alter any of the transcription factor binding sites when examined. The SNP variant in construct 3 (G/C \#rs3024944) is located in a transcription factor binding site TCF-1. The TCF-1 is a member of family of genes with homology to high mobility group I (HMG) proteins (Castrop et al. 1995). This family also includes lymphocyte enhancer-binding factor-1 and SRY-related HMG-box (SOX-4). TCF-1, in part with $\beta$-catenin, plays a pivotal role in $\mathrm{T}$ cell activation and is specifically expressed in T lymphocytes (Staal et al. 1999, Yu et al. 2010). Therefore individuals with this SNP variant (G/C \#rs3024944) may possibly have an elevated $\mathrm{T}$ cell response to infections.

STAT6 gene promoter variants had been previously shown to be associated with the outcome of several human diseases. A major association was inferred between a genetic variant (G/A 4219) of the 3'-UTR regulatory element of the STAT6 gene in an Ascaris infection study (Peisong et al. 2004). The study explicitly concluded that the homozygous state of this genetic variant indicated a strong association with diminished Ascaris burden in children living in an area of endemic infection. Another study concluded, however, that a promoter polymor- 
phism (rs324013) in STAT6 was found to be associated with the control of infection levels in urinary schistosomiasis (He et al. 2008). They also detected an additive effect between promoter polymorphisms rs324013 and rs1800925 in the STAT6 gene locus, and both these polymorphisms affected transcription factor binding when analyzed by an electrophoretic mobility shift assay. A very recent study revealed that constitutive STAT6 signalling in a Th cell impairs the suppressive potential of Tregs. The IL4-STAT6 signalling pathway plays a predominant role in $\mathrm{Th} 2$ cells proliferation and of Treg cells (Pillemer et al. 2009). The proliferation of naive T cells to Treg cells is regulated or kept in check by two factors, namely, transforming growth factor-beta (TGFß) and STAT6. TGFß when bound to the specific enhancer region of the FoxP3 promoter induces the conversion of naive T cells to Tregs, whereas STAT6 binding to a specific silencer region of FoxP3 promoter blocks the proliferation of naive $\mathrm{T}$ cells to Tregs (Takaki et al. 2008). When it comes to infection, the role of STAT6 remains vital in the control of the Treg population. A polymorphism in the STAT6 promoter that ably alters STAT6 expression can possibly increase proliferation of Tregs, thereby leading to a diffused immune response in the host with an elevated parasitic burden.

The identified regulatory SNPs in this current study will afford useful information for understanding the relevance of sequence polymorphisms in populations of different ethnic backgrounds and may serve as a basis to study parasite susceptibility in association studies. These regulatory polymorphisms in the promoter regions of STAT6 may assist in identifying potential targets for the therapeutic control of gene expression. In summary, we identified three SNP variants in the proximal promoter regions of the STAT6 gene by direct sequencing using DNA samples from 40 unrelated Gabonese individuals and investigated the functional activity of these promoter variants. The detection of such human immune gene polymorphisms will remain crucial in a genetic makeup of the patient population to level of infection and to that of expression of Treg activity.

\section{ACKNOWLEDGEMENTS}

To A Weierich, V Galinat and V Grummes, for technical assistance.

\section{REFERENCES}

Barrett JC, Fry B, Maller J, Daly MJ 2005. Haploview: analysis and visualization of LD and haplotype maps. Bioinformatics 21: 263-265.

Belkaid Y, Rouse BT 2005. Natural regulatory T cells in infectious disease. Nat Immunol 6: 353-360.

Castrop J, van Wichen D, Koomans-Bitter M, van de Wetering M, de Weger R, van Dongen J, Clevers H 1995. The human TCF-1 gene encodes a nuclear DNA-binding protein uniquely expressed in normal and neoplastic T-lineage lymphocytes. Blood 86: 3050-3059.

He H, Isnard A, Kouriba B, Cabantous S, Dessein A, Doumbo O, Chevillard C 2008. A STAT6 gene polymorphism is associated with high infection levels in urinary schistosomiasis. Genes Immun 9: 195-206.

Hromas R, Collins SJ, Hickstein D, Raskind W, Deaven LL, O'Hara P, Hagen FS, Kaushansky K 1991. A retinoic acid-responsive human zinc finger gene, MZF-1, preferentially expressed in myeloid cells. J Biol Chem 266: 14183-14187.

Jüliger S, Bongartz M, Luty AJ, Kremsner PG, Kun JF 2003. Functional analysis of a promoter variant of the gene encoding the interferon-gamma receptor chain I. Immunogenetics 54: 675-680.

Kaplan MH, Schindler U, Smiley ST, Grusby MJ 1996. STAT6 is required for mediating responses to IL-4 and for development of Th2 cells. Immunity 4: 313-319.

Maizels RM 2009. Parasite immunomodulation and polymorphisms of the immune system. $J$ Biol 8: 62 .

Morris JF, Rauscher FJ 3rd, Davis B, Klemsz M, Xu D, Tenen D, Hromas R 1995. The myeloid zinc finger gene, MZF-1, regulates the CD34 promoter in vitro. Blood 86: 3640-3647.

Peisong G, Yamasaki A, Mao XQ, Enomoto T, Feng Z, Gloria-Bottini F, Bottini E, Shirakawa T, Sun D, Hopkin JM 2004. An asthmaassociated genetic variant of STAT6 predicts low burden of ascaris worm infestation. Genes Immun 5: 58-62.

Pillemer BB, Qi Z, Melgert B, Oriss TB, Ray P, Ray A 2009. STAT6 activation confers upon $\mathrm{T}$ helper cells resistance to suppression by regulatory T cells. J Immunol 183: 155-163.

Rozen S, Skaletsky H 2000. Primer3 on the www for general users and for biologist programmers. Methods Mol Biol 132: 365-386.

Sakaguchi S 2000. Regulatory T cells: key controllers of immunologic self-tolerance. Cell 101: 455-458.

Sakaguchi S 2004. Naturally arising $\mathrm{CD}^{+}$regulatory T cells for immunologic self-tolerance and negative control of immune responses. Аnпu Rev Immunol 22: 531-562.

Sakaguchi S 2005. Naturally arising Foxp3-expressing CD25+ CD4 ${ }^{+}$ regulatory $\mathrm{T}$ cells in immunological tolerance to self and nonself. Nat Immunol 6: 345-352.

Schindler C, Darnell JE Jr 1995. Transcriptional responses to polypeptide ligands: the JAK-STAT pathway. Annu Rev Biochem 64: 621-651.

Staal FJ, Burgering BM, van de Wetering M, Clevers HC 1999. Tcf1-mediated transcription in T lymphocytes: differential role for glycogen synthase kinase-3 in fibroblasts and T cells. Int Immunol 11: 317-323.

Takaki H, Ichiyama K, Koga K, Chinen T, Takaesu G, Sugiyama Y, Kato S, Yoshimura A, Kobayashi T 2008. STAT6 inhibits TGFbeta1-mediated Foxp3 induction through direct binding to the Foxp3 promoter, which is reverted by retinoic acid receptor. J Biol Chem 283: 14955-14962.

Yu Q, Sharma A, Sen JM 2010. TCF1 and beta-catenin regulate T cell development and function. Immunol Res 47: 45-55. 